\begin{tabular}{|c|l|}
\hline Title & DOCK2 regulates cell proliferation through Rac and ERK activation in B cell lymphoma \\
\hline Author(s) & $\begin{array}{l}\text { Wang, Lei; Nishihara, Hiroshi; Kimura, Taichi; Kato, Y asutaka; Tanino, Mishie; Nishio, Mitsufumi; Obara, Masato; } \\
\text { Endo, Tomoyuki; Koike, Takao; Tanaka, Shinya }\end{array}$ \\
\hline Citation & $\begin{array}{l}\text { Biochemical and Biophysical Research Communications, 395(1), 111-115 } \\
\text { https://doi.org/10.1016/.bbrc.2010.03.148 }\end{array}$ \\
\hline Issue Date & 2010-0423 \\
\hline Doc URL & http://hdl.handle.net/2115/43094 \\
\hline Type & article (author version) \\
\hline File Information & BBRC395-1_111-115.pdf \\
\hline
\end{tabular}

Instructions for use 


\section{DOCK2 regulates cell proliferation through Rac and ERK activation}

\section{in $B$ cell lymphoma.}

Lei Wang ${ }^{\mathrm{a}}$, Hiroshi Nishihara ${ }^{\mathrm{b} *}$, Taichi Kimura ${ }^{\mathrm{a}}$, Yasutaka Kato ${ }^{\mathrm{a}}$, Mishie Tanino ${ }^{\mathrm{a}}$,

Mitsufumi Nishio ${ }^{c}$, Masato Obara ${ }^{c}$, Tomoyuki Endo ${ }^{c}$, Takao Koike ${ }^{c}$, and Shinya Tanaka ${ }^{a}$

${ }^{a}$ Laboratory of Cancer Research and ${ }^{\mathrm{b}}$ Laboratory of Translational Pathology,

Department of Pathology, and ${ }^{\mathrm{c}}$ Department of Internal Medicine II, Hokkaido

University Graduate School of Medicine, N15W7, Kitaku, Sapporo, 060-8638, Japan;

* Corresponding Author: FAX: +81-11-706-5902,

e-mail: nisihara@patho2.med.hokudai.ac.jp 


\section{Abstract}

DOCK2; a member of the CDM protein family, regulates cell motility and cytokine production through the activation of Rac in mammalian hematopoietic cells and plays a pivotal role in the modulation of the immune system. Here we demonstrated the alternative function of DOCK2 in hematopoietic tumor cells, especially in terms of its association with the tumor progression.

Immunostaining for DOCK2 in 20 cases of human B cell lymphoma tissue specimens including diffuse large B cell lymphoma and follicular lymphoma revealed the prominent expression of DOCK2 in all of the lymphoma cells. DOCK2-knockdown (KD) of the B cell lymphoma cell lines, Ramos and Raji, using the lentiviral shRNA system presented decreased cell proliferation compared to the control cells. Furthermore, the tumor formation of DOCK2-KD Ramos cell in nude mice was significantly abrogated. Western blotting analysis and pull-down assay using GST-PAK-RBD kimeric protein suggested the presence of DOCK2-Rac-ERK pathway regulating the cell proliferation of these lymphoma cells. This is the first report to clarify the prominent role of DOCK2 in hematopoietic malignancy. 
Keywords: DOCK2, B cell lymphoma, Rac, ERK, hematopoietic malignancy 


\section{Introduction}

A guanine nucleotide exchange factor (GEF), DOCK2, which is a hematopoietic cell-specific homolog of the CDM family protein[1], plays an essential role in the regulation of cell motility and cytokine production through the activation of Rac in human hematopoietic cells [2; 3; 4]. A knockout study against DOCK2 demonstrated the alternative role of DOCK2, regarding murine T- and B-cell motility [5] and control of the cardiac transplant rejection in mice [6]. The prominent roles of DOCK2, such as immune modulation associated with $\mathrm{T}$ cell differentiation [7; 8] and neutrophil chemotaxis $[9 ; 10]$ were also disclosed.

A small GTPase Rac, a member of Rho protein family, regulates the cell motility and cell cycle through the reorganization of the actin cytoskeleton [11]. The activators for Rac, such as Vav and Tiam-1, have been reported to associate with tumor invasion and metastasis in several non-hematopoietic malignancies [12; 13; 14], although the distinctive role of the DOCK2-Rac pathway in tumorigenesis remains to be investigated. In this study, we have explored the alternative function of DOCK2 associated with carcinogenesis especially in the hematopoietic malignancy. 


\section{Materials and Methods}

\section{Histological analysis and immunohistochemistry}

Formalin-fixed, paraffin-embedded human malignant lymphoma specimens which included 10 cases each of diffuse large B cell lymphoma and follicular lymphoma were selected. The pathological diagnosis of all 20 cases was made in our department and an affiliated hospital. The human specimens and the formalin-fixed paraffin-embedded xenografts removed from nude mice were sectioned and stained with hematoxylin and eosin (HE) using standard protocol. Immunohistochemistry was performed using anti-DOCK2 [2] and anti-Ki67 (MIB1; Dako, Glostrup, Denmark) antibodies.

\section{Cell lines}

The human B cell lymphoma cell line, Ramos and Raji cell line, was obtained from RIKEN BioResource Resource Center Cell Bank (Tsukuba, Japan). Cells were maintained in Dulbecco's modified minimal essential medium (DMEM) supplemented with 10\% fetal bovine serum (FBS), $2 \mathrm{mM} \mathrm{L-glutamine,} \mathrm{and} 100 \mathrm{U} / \mathrm{ml}$ penicillin and streptomycin. 
To measure growth rates of the cells, 1 x $10^{5}$ cells were seeded onto 60 -mm diameter plates with DMEM containing 2\% FBS, and the numbers of cells were counted every day using a hemocytometer (Fisher Scientific, Pittsburgh, PA).

\section{Establishment of DOCK2 KD malignant lymphoma cell lines}

We have employed BLOCK-iT ${ }^{\mathrm{TM}}$ Lentiviral miR RNAi Expression System (Invitrogen, Carlsbad, CA) to knock down the DOCK2 expression in lymphoma cells. The target sequences (codons 594- and 1364- ) were determined by BLOCK-iT ${ }^{\mathrm{TM}}$ RNAi Designer (Invitrogen), and oligonucleotides were subcloned into the pLenti6/V5-DEST

Gateway® Vector. ${ }^{\circledR}$ pcDNA ${ }^{\mathrm{TM}}$ 6.2-GW/EmGFP-miR-neg control (Invitrogen) was used as a negative control which contained random oligonucleotide. The lentiviruses which contained short hairpin RNA were obtained following the manufacturer's protocol and were transduced into the Ramos and Raji lymphoma cells. Cells were cultured in the presence of $20 \mu \mathrm{g} / \mathrm{ml}$ blasticidin (invitrogen), and drug-resistant clones were isolated.

\section{SDS-PAGE and immunoblotting}

SDS-PAGE and immunoblotting were performed as described previously [15].

Antibodies were obtained from the following sources: anti-phospho-ERK, 
anti-phospho-AKT, anti-phospho-p38 (Cell Signaling Technology, USA); anti-Actin

antibody (Chemicon International, Temecula, CA); anti-Rac1 (Transduction

Laboratories, KY).

Pull-down assay for Rac1 activity

Detection of the GTP-bound form of Rac1 was performed as described previously [3].

Briefly, cells were lysed with buffer containing 25 mM HEPES (pH 7.4), 150 mM NaCl, 10\% glycerol, 1 mM EDTA, 1\% NP40, 10 mM MgCl $2,1 \mu \mathrm{g} / \mathrm{ml}$ aprotinin and $1 \mathrm{mM}$

PMSF. Lysates were centrifuged at 12000 r.p.m. at $4{ }^{\circ} \mathrm{C}$ for 1 min, and the supernatants were incubated with $10 \mu \mathrm{g}$ of purified GST-PAK2-RBD and glutathione-Sepharose 4B beads. The proteins bound to GST fusion protein were separated by SDS-PAGE and analysed by immunoblotting with anti-Rac1 antibody.

\section{Reagents}

A phosphatidylinositol-3 kinase (PI3K) inhibitor,LY294002; and Rac specific inhibitor, NSC23766 were obtained from Calbiochem (San Diego, CA), and MAPK/ERK kinase (MEK) inhibitor, U0126 was from Cell Signaling Technology (Beverly, MA).

\section{Xenograft propagation.}


Subcutaneously, 1 x $10^{6}$ cells were injected into four of the 3-week-old female nude mice, BALB/cA Jcl-nu (nu/nu) (CLEA Japan Inc., Japan). The mice were euthanized 18-21 days after injection, and tumors were removed and weighed. Histopathological examinations were performed, including hematoxylin and eosin stain and Ki-67 stain. 


\section{Results and Discussion}

DOCK2 expresses in human B cell lymphoma

The expression of DOCK2 was previously shown in several hematopoietic cell lines derived from human malignant lymphoma, such as Ramos from B cell lymphoma, as well as normal lymphocytes in human tissue specimens [2], although the distinctive role of the DOCK2-Rac pathway in tumorigenesis remains to be investigated. To confirm the expression of DOCK2 in human pathological tissue of hematopoietic malignancy, we performed immunostaining for DOCK2 using 20 cases of human lymphoma specimens. As a result, we observed the membraneous and cytoplasmic stain of DOCK2 in all lymphoma cases which we examined, including the following two common types of B cell lymphoma: diffuse large B cell lymphoma and follicular lymphoma (Fig. 1). The intensity of DOCK2 staining was similar to that of normal lymphocytes, suggesting that DOCK2 would be an essential molecule for tumor cells as well as non-tumorous lymphocytes.

DOCK2 regulates cell proliferation through Rac-ERK activation in human $B$ cell

\section{lymphoma in vitro}


To explore the role of DOCK2 in tumor progression, we have established DOCK2-KD

B cell lymphoma cell lines. We obtained the two types of B cell lymphoma, Ramos and Raji, in which the expression of DOCK2 was explicitly abrogated by the two types of shRNA targeting codon 594-, and codon 1364- (Fig. 2A). We also confirmed that the active form of Rac1, a specific effecter molecule for DOCK2, was significantly decreased in these DOCK2-KD cells (Fig. 2B). To assess the role of DOCK2 in growth response, we obtained the growth curve of these cells, and found that the growth rates of DOCK2-KD Ramos and Raji were significantly declined compare to the control cells (Fig. 3A). A specific Rac inhibitor, NSC23766, also inhibited the cell proliferation of wild type Ramos cells (Fig. 3B); therefore, we hypothesized whether the DOCK2-Rac pathway could influence some kinases associated with cell proliferation, such as MAP kinases and AKT [16]. In fact, western blotting analysis revealed the decreased phosphorylation level of ERK in DOCK2-KD Ramos cells (Fig. 2C) as well as in DOCK2-KD Raji cells (data not shown), while those of AKT and p38 were not altered (Fig. 2C). In addition, the result that NSC23766 significantly decreased the phosphorylation level of ERK in wild type Ramos cells (Fig. 2D) led us to conclude that 
the DOCK2-Rac-ERK signaling pathway is responsible for cell proliferation of B cell lymphoma. Although the Ras-ERK is one of the prominent downstream pathways of tyrosine kinase receptors such as EGFR for cell growth in most cancer cells, we observed no significant suppression of Ras activity in DOCK2-KD Ramos cells by pull down assay using GST-RafRBD (data not shown).

\section{DOCK2 regulates xenograft propagation of human B cell lymphoma in vivo}

We finally examined the in vivo tumorigenesis assay using DOCK2-KD Ramos cells.

The size of subcutaneous xenografts of DOCK2-KD Ramos cells was significantly smaller than that of control Ramos cells in 4 individual experiments (Fig. 4A) $(p<0.01)$.

Histopathological examinations confirmed that the tumor cells of all xenografts exhibited similar appearance in HE staining (Fig. 4C), and that the CD20-positive, CD3-negative immunophenotype was consistent with that of human B cell lymphoma (data not shown), while lower Mib-1 labeling indices were observed in all of the 4 xenografts of DOCK2-KD Ramos cells (Fig. 4C). We also obtained a similar result using DOCK2-KD Raji cells (data not shown). 
Here we elucidated the alternative role of DOCK2, especially in the tumor progression of B cell lymphoma and the significance of the pathway of DOCK2-Rac-ERK in lymphoma cell proliferation. Our conclusion is consistent with the recent findings which demonstrated the presence of the DOCK2-Rac-MEK-ERK pathway in mouse $\mathrm{CD}^{+} \mathrm{T}$ cells [7], although the significance of the conventional Rac-PAK pathway in cancer progression has been implicated [17].

The GTPase Rac1 is a key molecule for transformation driven by oncogenic Ras through the cytoskeletal reorganization [18]. The activation of Rac1 by Tiam-1 was described to be associated with tumor progression in T cell lymphoma [19], and Rac1 was also reported to inhibit apoptosis in the human lymphoma cells through the activation of Bad phosphorylation [20]. Unlike what was determined for oncogene Rac, very few activating mutations are identified in the gene encoding Rac; therefore, the activation of the Rac-specific GEFs such as Tiam-1 and DOCK2 might be a crucial event in the progression of hematopoietic malignancy. Our results suggest the possible target molecules of DOCK2 and Rac for lymphoma therapy, although the detailed regulation mechanism of DOCK2 and Rac has to be explored, and clinical 
manifestations of the expression of DOCK2 and Rac in large numbers of lymphoma samples are essential. 


\section{Acknowledgement}

This work was supported in part by a grant from a Grant-in-aid from the Ministry of

Education, Science, Technology, Sports and Culture of Japan. 


\section{Figure Legends}

\section{Fig. 1 DOCK2 expressed in human B cell lymphoma tissues}

HE staining revealed the diffuse infiltration of enlarged atypical lymphocytes (Cases 1 and 2; diffuse large B cell lymphoma) or vague nodular lesion consist of small atypical lymphocytes (Case 3 and 4; follicular lymphoma). Immunostaining represented the distinct expression of DOCK2 in all of the lymphoma cells as well as reactive lymphoid cells, while no expression was observed in vascular endothelial cells or other non-hematopoietic tissues. Asterisks indicate the vascular lumen. Scale bars mean $100 \mu \mathrm{m}$ in the left lane or $0.5 \mu \mathrm{m}$ in the two right lanes, respectively. Squares indicate the areas subjected to higher magnification.

Fig. 2 The levels of GTP-Rac and phospho-ERK were decreased in DOCK2-knockdown lymphoma cells

The expression of DOCK2 and the phosphorylation of several kinases in shRNA-transduced Ramos (A, C) and Raji (A) cells were analyzed by immunoblotting. Control, randomized shRNA; 594 and1364, shRNA designated for codons 594- and 
1364- of DOCK2 cDNA, respectively. (B) Pulldown analysis for GTP-Rac1 using GST-PAK2-RBD was performed in shRNA-transduced Ramos and Raji cells. (D) The wild type Ramos cells were cultured in the presence of the indicated inhibitors at $50 \mu \mathrm{M}$ for 48 hrs and applied for immunoblotting analysis. LY294002, PI3K inhibitor; U0126, MEK inhibitor; NSC23766, Rac inhibitor.

Fig. 3 DOCK2 and Rac contribute growth properties of lymphoma cells in vitro The shRNA-transduced Ramos and Raji cells (A), and the wild type Ramos cells with or without NSC23766 $(50 \mu \mathrm{M})$ were plated onto $60-\mathrm{mm}$ diameter plates and the numbers of cells were counted every day using a hemocytometer. Results are shown as mean \pm S.D. of at least 3 samples each. For statistical comparisons between groups, the Student's $t$ test was used.

Fig. 4 DOCK2 is responsible for tumor formation of the lymphoma cells in nude mice The shRNA-transduced Ramos cells were infected into the nude mice subcutaneously. After 18-21 days, the tumors were removed from the mice and applied for 
histopathological examinations. (A) Examples of macroscopical appearance of removed tumors are shown. (B) The weight of the removed tumors from each mouse $(n=4)$ is demonstrated by dot plot. These results were statistically analyzed by Student's $t$ test. (C) Formalin-fixed, paraffin-embedded removed tumors were applied for HE staining (top) and immunohistochemistry with anti-Ki67 antibody (Mib-1; bottom). Mib-1 labeling index (Mib-1 LI) is indicated as mean \pm S.D. of 3 high powered fields for each tumor, which was statistically significant ( $\mathrm{p}<0.01)$. 


\section{References}

[1] J.F. Cote, and K. Vuori, Identification of an evolutionarily conserved superfamily of DOCK180-related proteins with guanine nucleotide exchange activity. J Cell Sci 115 (2002) 4901-13.

[2] H. Nishihara, S. Kobayashi, Y. Hashimoto, F. Ohba, N. Mochizuki, T. Kurata, K. Nagashima, and M. Matsuda, Non-adherent cell-specific expression of DOCK2, a member of the human CDM-family proteins. Biochim Biophys Acta 1452 (1999) 179-87.

[3] H. Nishihara, M. Maeda, A. Oda, M. Tsuda, H. Sawa, K. Nagashima, and S. Tanaka, DOCK2 associates with CrkL and regulates Rac1 in human leukemia cell lines. Blood 100 (2002) 3968-74.

[4] H. Nishihara, M. Maeda, M. Tsuda, Y. Makino, H. Sawa, K. Nagashima, and S. Tanaka, DOCK2 mediates T cell receptor-induced activation of Rac2 and IL-2 transcription. Biochem Biophys Res Commun 296 (2002) 716-20.

[5] Y. Fukui, O. Hashimoto, T. Sanui, T. Oono, H. Koga, M. Abe, A. Inayoshi, M. Noda, M. Oike, T. Shirai, and T. Sasazuki, Haematopoietic cell-specific CDM family protein DOCK2 is essential for lymphocyte migration. Nature 412 (2001) 826-31.

[6] H. Jiang, F. Pan, L.M. Erickson, M.S. Jang, T. Sanui, Y. Kunisaki, T. Sasazuki, M. Kobayashi, and Y. Fukui, Deletion of DOCK2, a regulator of the actin cytoskeleton in lymphocytes, suppresses cardiac allograft rejection. J Exp Med 202 (2005) 1121-30.

[7] K. Gollmer, F. Asperti-Boursin, Y. Tanaka, K. Okkenhaug, B. Vanhaesebroeck, J.R. Peterson, Y. Fukui, E. Donnadieu, and J.V. Stein, CCL21 mediates CD4+ T-cell costimulation via a DOCK2/Rac-dependent pathway. Blood 114 (2009) 580-8.

[8] Y. Tanaka, S. Hamano, K. Gotoh, Y. Murata, Y. Kunisaki, A. Nishikimi, R. Takii, M. Kawaguchi, A. Inayoshi, S. Masuko, K. Himeno, T. Sasazuki, and Y. Fukui, T helper type 2 differentiation and intracellular trafficking of the interleukin 4 receptor-alpha subunit controlled by the Rac activator Dock2. Nat Immunol 8 (2007) 1067-75.

[9] A. Nishikimi, H. Fukuhara, W. Su, T. Hongu, S. Takasuga, H. Mihara, Q. Cao, F. Sanematsu, M. Kanai, H. Hasegawa, Y. Tanaka, M. Shibasaki, Y. Kanaho, T. 
Sasaki, M.A. Frohman, and Y. Fukui, Sequential regulation of DOCK2 dynamics by two phospholipids during neutrophil chemotaxis. Science 324 (2009) 384-7.

[10] Y. Kunisaki, A. Nishikimi, Y. Tanaka, R. Takii, M. Noda, A. Inayoshi, K. Watanabe, F. Sanematsu, T. Sasazuki, T. Sasaki, and Y. Fukui, DOCK2 is a Rac activator that regulates motility and polarity during neutrophil chemotaxis. J Cell Biol 174 (2006) 647-52.

[11] S. Etienne-Manneville, and A. Hall, Rho GTPases in cell biology. Nature 420 (2002) 629-35.

[12] P.S. Steeg, Metastasis suppressors alter the signal transduction of cancer cells. Nat Rev Cancer 3 (2003) 55-63.

[13] G. Lazer, Y. Idelchuk, V. Schapira, E. Pikarsky, and S. Katzav, The haematopoietic specific signal transducer Vav1 is aberrantly expressed in lung cancer and plays a role in tumourigenesis. J Pathol 219 (2009) 25-34.

[14] M.E. Minard, L.S. Kim, J.E. Price, and G.E. Gallick, The role of the guanine nucleotide exchange factor Tiam1 in cellular migration, invasion, adhesion and tumor progression. Breast Cancer Res Treat 84 (2004) 21-32.

[15] L. Wang, K. Tabu, T. Kimura, M. Tsuda, H. Linghu, M. Tanino, S. Kaneko, H. Nishihara, and S. Tanaka, Signaling adaptor protein Crk is indispensable for malignant feature of glioblastoma cell line KMG4. Biochem Biophys Res Commun 362 (2007) 976-81.

[16] J.A. McCubrey, L.S. Steelman, W.H. Chappell, S.L. Abrams, E.W. Wong, F. Chang, B. Lehmann, D.M. Terrian, M. Milella, A. Tafuri, F. Stivala, M. Libra, J. Basecke, C. Evangelisti, A.M. Martelli, and R.A. Franklin, Roles of the Raf/MEK/ERK pathway in cell growth, malignant transformation and drug resistance. Biochim Biophys Acta 1773 (2007) 1263-84.

[17] R.K. Vadlamudi, and R. Kumar, P21-activated kinases in human cancer. Cancer Metastasis Rev 22 (2003) 385-93.

[18] R.G. Qiu, J. Chen, D. Kirn, F. McCormick, and M. Symons, An essential role for Rac in Ras transformation. Nature 374 (1995) 457-9.

[19] K. Strumane, J.Y. Song, I. Baas, and J.G. Collard, Increased Rac activity is required for the progression of T-lymphomas induced by Pten-deficiency. Leuk Res 32 (2008) 113-20. 
[20] B. Zhang, Y. Zhang, and E. Shacter, Rac1 inhibits apoptosis in human lymphoma cells by stimulating Bad phosphorylation on Ser-75. Mol Cell Biol 24 (2004) 6205-14. 


\section{Fig. 1}

HE x20

Case 1

Case 2
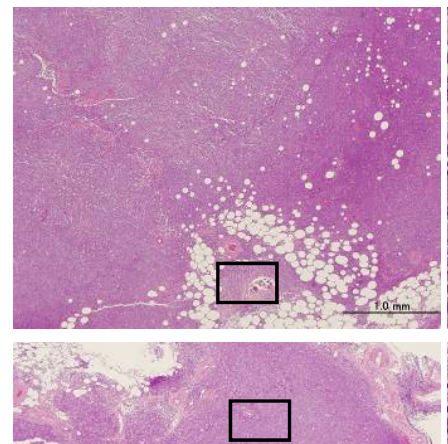

Case 3

Case 4

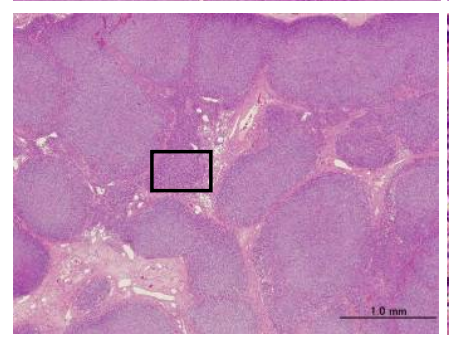

HE x400
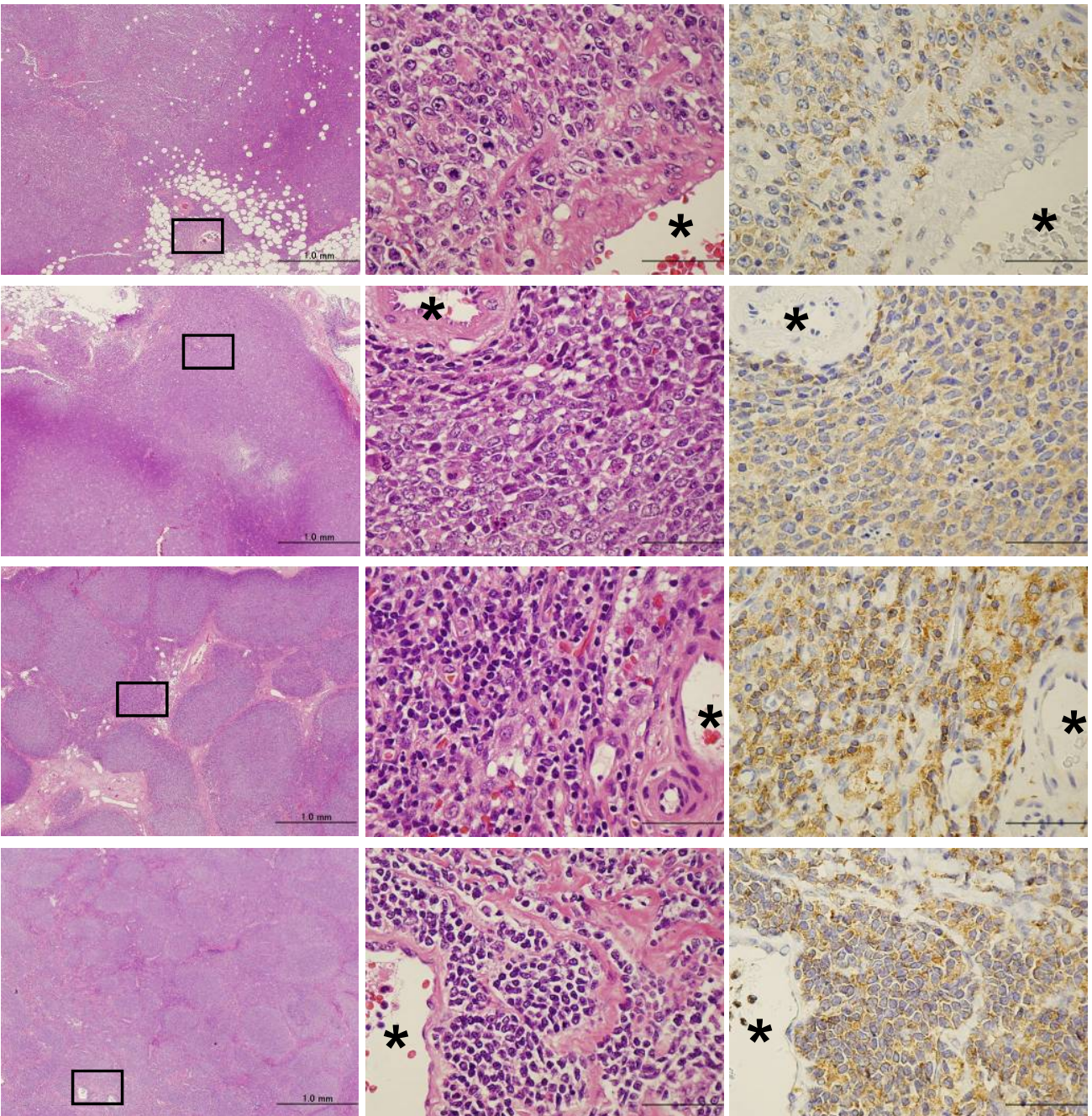

DOCK2 x400
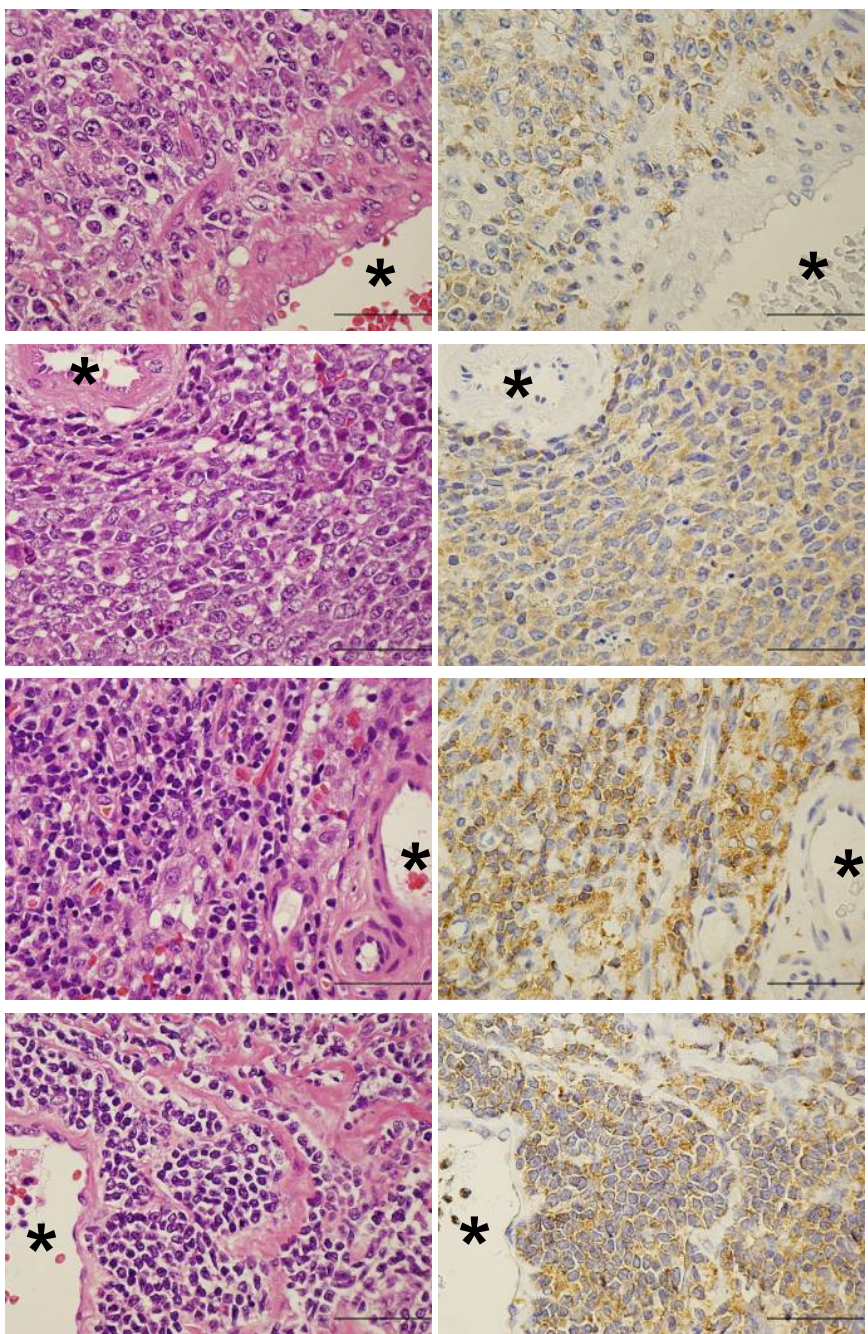
Fig. 2

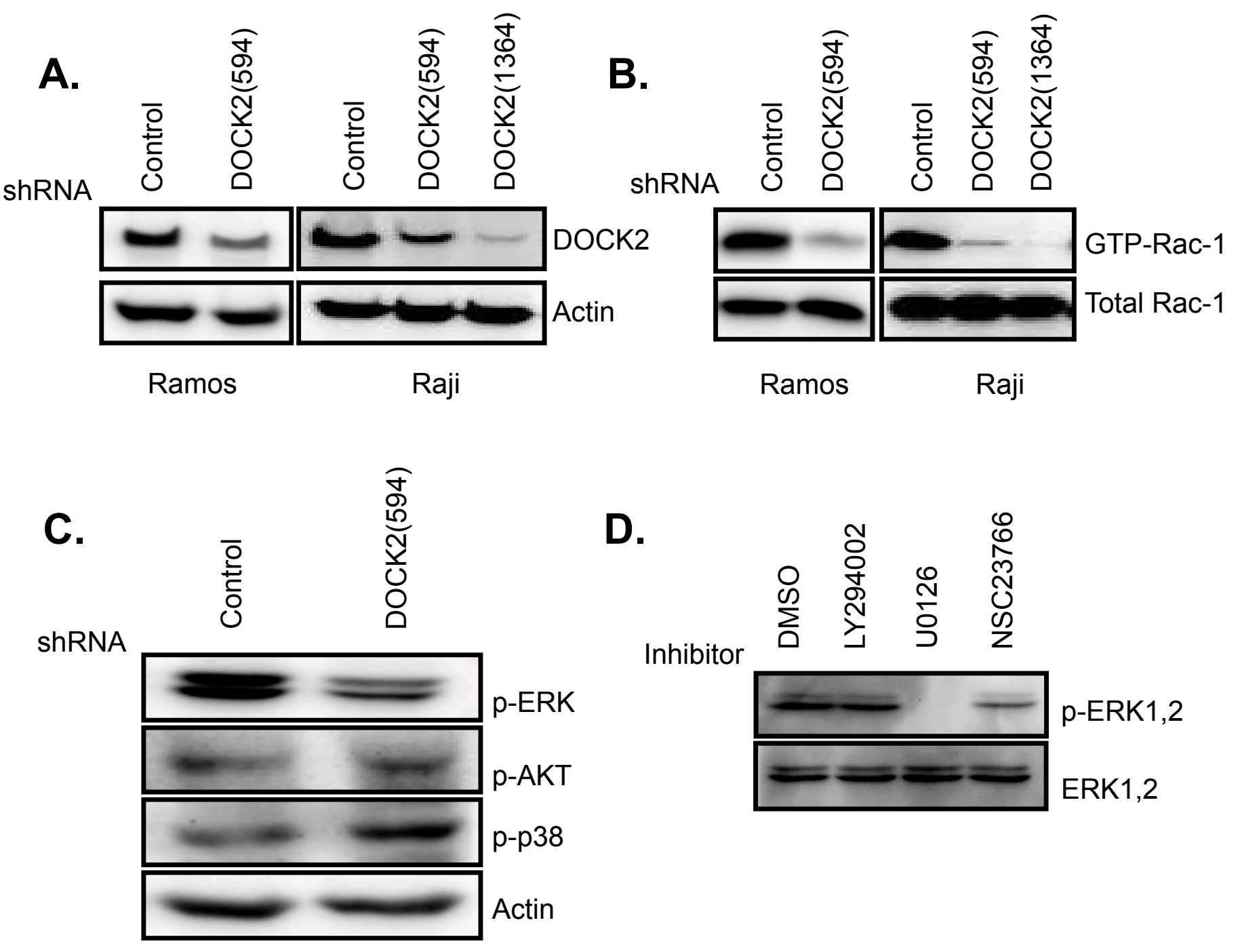


A.
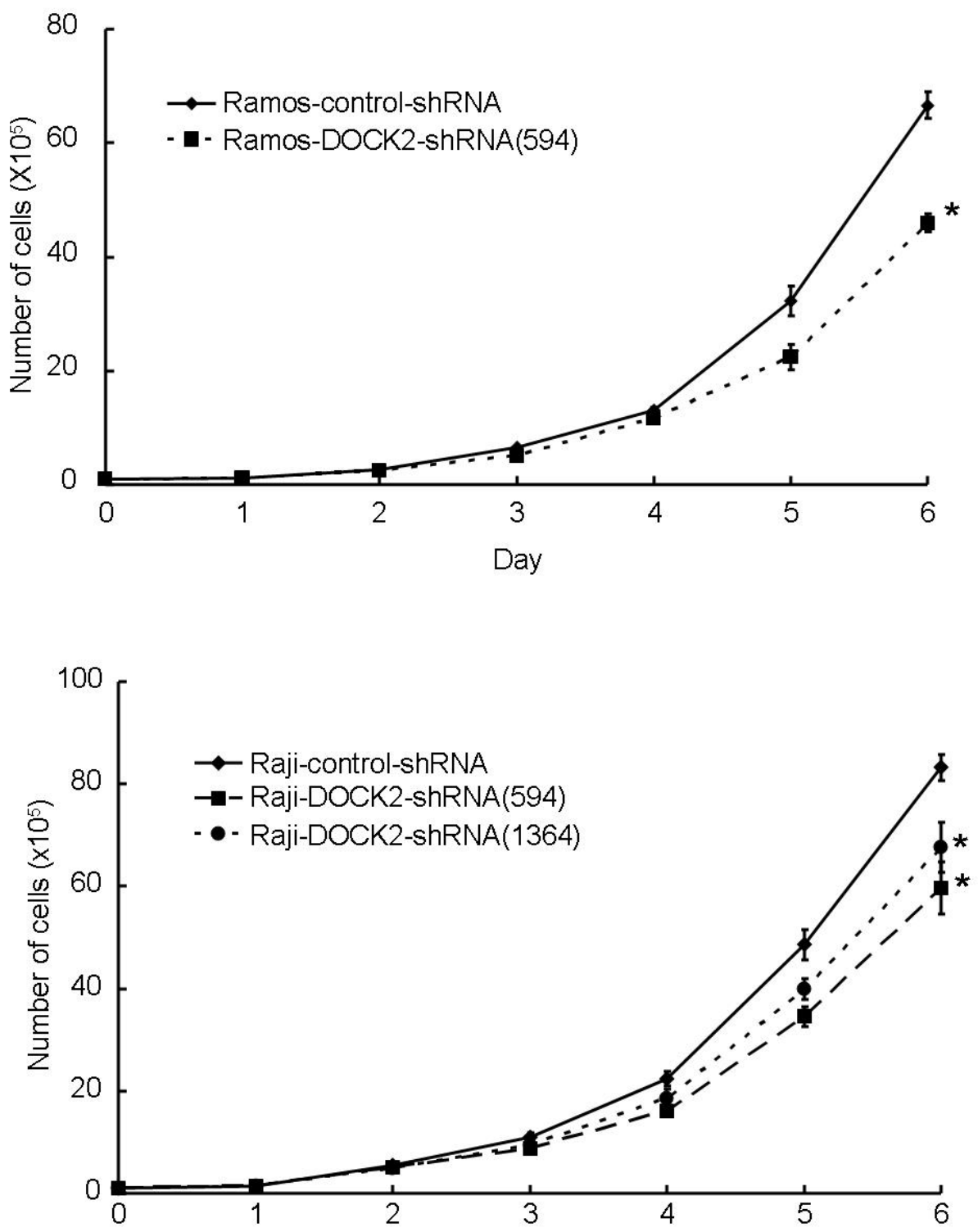

B.

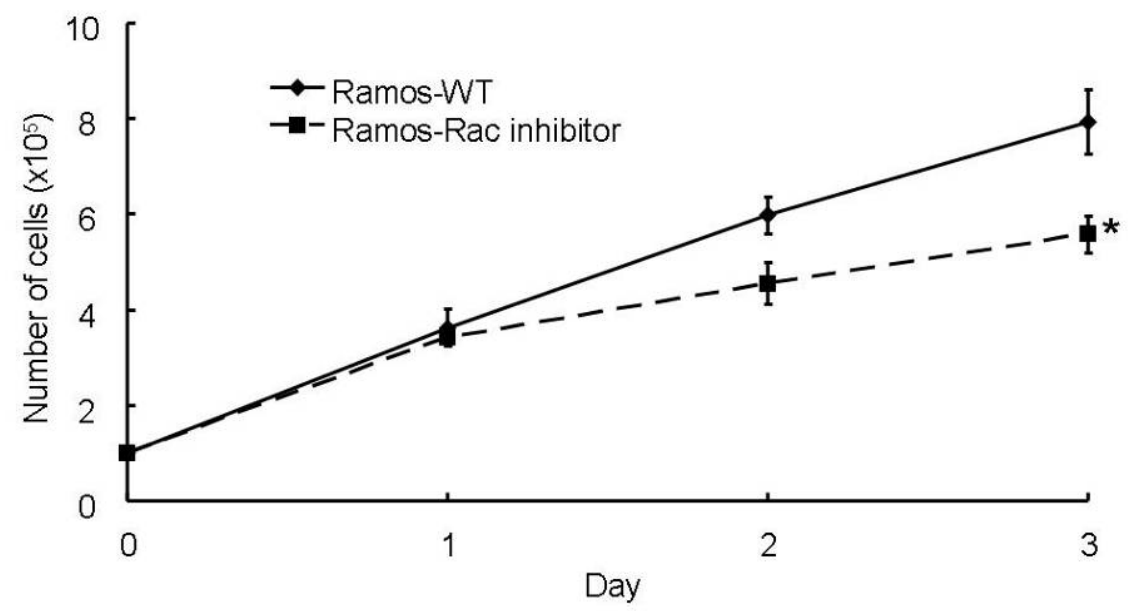


Fig. 4

A. B

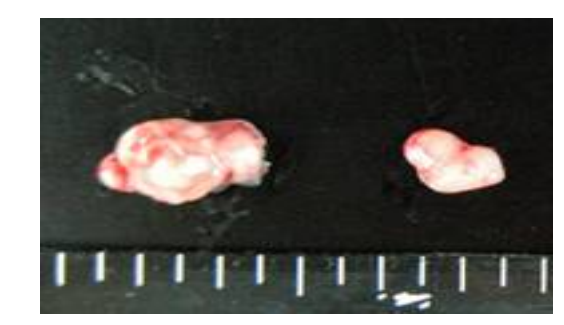

Control-shRNA DOCK2shRNA(594)

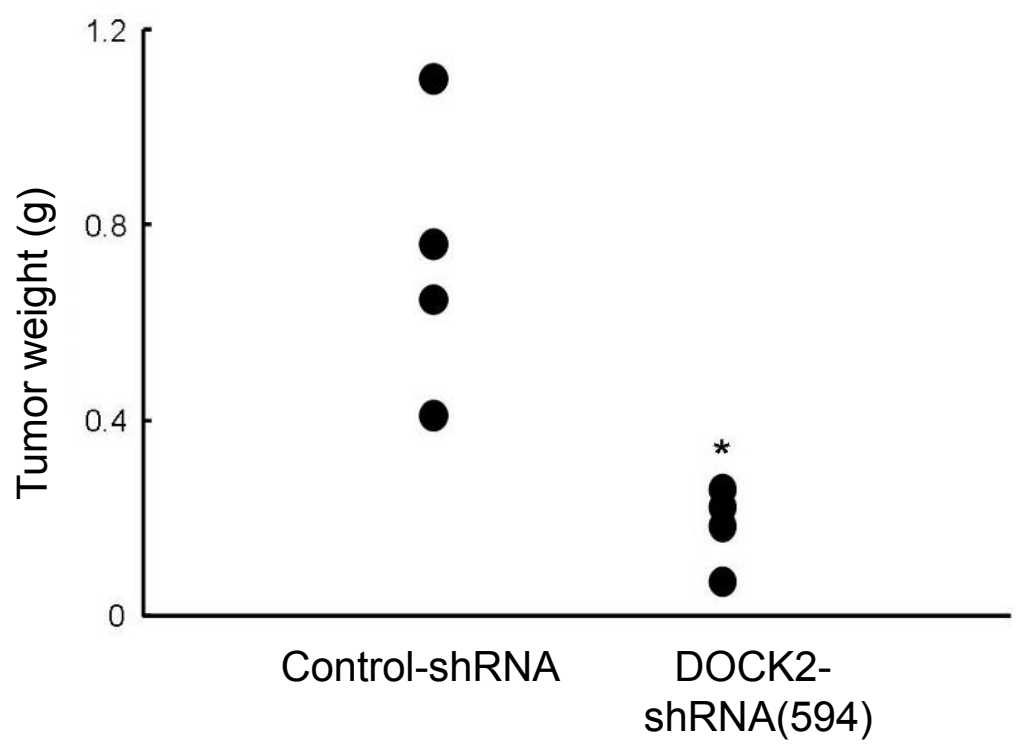

C.

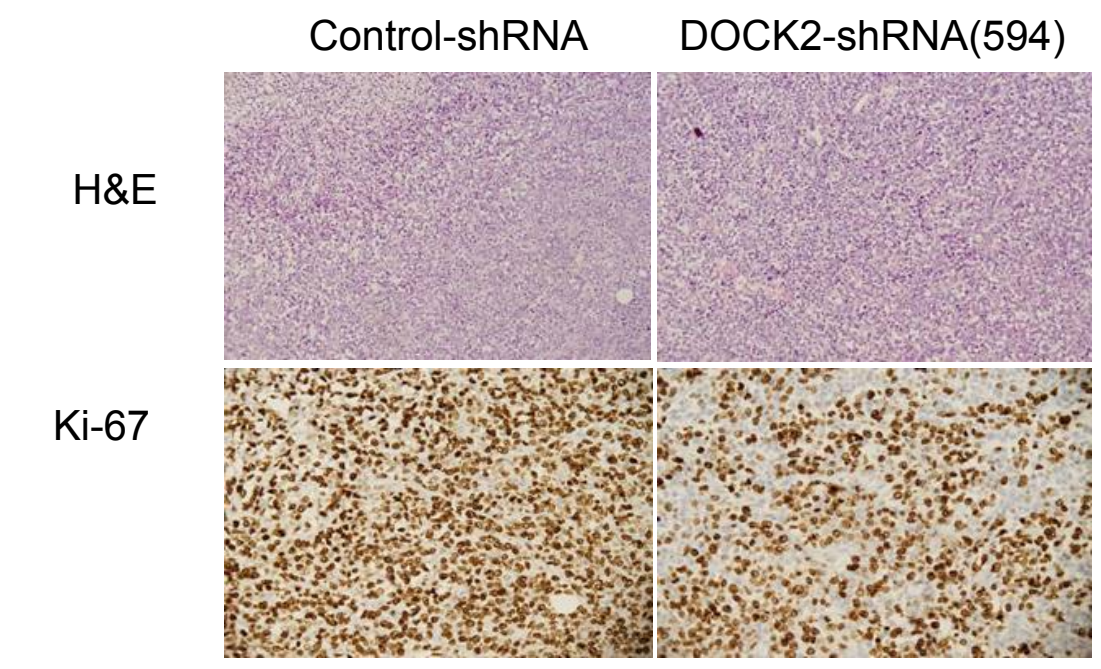

Mib-1 LI (\%) $\quad 71 \pm 9.3 \quad 53 \pm 4.4$ 